\title{
Case Report \\ Complications Following Masseteric Nerve Neurectomy with Radiofrequency for the Treatment of Temporomandibular Disorders-A Case Series and Literature Review
}

\author{
Il-San Cho ${ }^{1,2}$, Jung Hwan Jo ${ }^{1,2}$ (D) and Ji Woon Park $1,2,3, * \mathbb{D}$ \\ 1 Department of Oral Medicine and Oral Diagnosis, School of Dentistry, Seoul National University, \\ Seoul 03080, Korea; s991320@naver.com (I.-S.C.); junghwanjo@snu.ac.kr (J.H.J.) \\ 2 Department of Oral Medicine, Seoul National University Dental Hospital, Seoul 03080, Korea \\ 3 Dental Research Institute, Seoul National University, Seoul 03080, Korea \\ * Correspondence: ankara01@snu.ac.kr; Tel.: +82-2-2072-4912
}

Citation: Cho, I.-S.; Jo, J.H.; Park, J.W. Complications Following Masseteric

Nerve Neurectomy with

Radiofrequency for the Treatment of Temporomandibular Disorders-A Case Series and Literature Review. Appl. Sci. 2021, 11, 5824. https:// doi.org/10.3390/app11135824

Academic Editor: Francesco Cappello

Received: 8 June 2021

Accepted: 20 June 2021

Published: 23 June 2021

Publisher's Note: MDPI stays neutral with regard to jurisdictional claims in published maps and institutional affiliations.

Copyright: () 2021 by the authors. Licensee MDPI, Basel, Switzerland. This article is an open access article distributed under the terms and conditions of the Creative Commons Attribution (CC BY) license (https:// creativecommons.org/licenses/by/ $4.0 /)$.

\begin{abstract}
This article describes two cases of masseteric nerve neurectomy with radiofrequency done with the intention to treat temporomandibular disorders and related symptoms; the patients then visited our clinic complaining of side-effects after the procedure. A literature review was conducted to find scientific evidence relevant to masseteric nerve neurectomy with radiofrequency. A 21-year-old male patient visited with the chief complaint of swelling of both cheeks, dizziness, and generalized lethargy occurring after masseteric nerve neurectomy using radiofrequency. His mouth opening range was restricted. Magnetic resonance imaging indicated post-procedural inflammation with hemorrhage within both masseter muscles. A 28-year-old male patient visited with the chief complaint of occlusal discomfort and disocclusion after masseteric nerve neurectomy using radiofrequency. His occlusion was abnormal with only both second molars occluding. Overbite was $-1 \mathrm{~mm}$. Cone-beam computed tomography indicated degenerative joint disease of both condyles. In case 1, pharmacotherapy and physical therapy relieved overall symptoms. In case 2, although exacerbation of symptoms repeatedly occurred, long-term stabilization splint and physical therapy alleviated the temporomandibular disorders symptoms. However, the occlusion remained unstable. Scientific evidence of masseteric nerve neurectomy using radiofrequency for the treatment of temporomandibular disorders is still lacking. Therefore, conservative treatment should remain as the first line approach for temporomandibular disorders.
\end{abstract}

Keywords: temporomandibular disorders; masseteric nerve; denervation; masseter muscle; volume reduction; radiofrequency therapy; side effects

\section{Introduction}

The application of radiofrequency neurectomy on the masseteric nerve has steadily increased and is now widely used for cosmetic purposes such as reduction of masseter muscle hypertrophy. Furthermore, attempts have been made to apply masseteric nerve neurectomy for the treatment of temporomandibular disorders (TMD). As the number of cases grow, related side-effects and medical disputes are also increasing [1].

Initially, radiofrequency neurectomy was developed as a method to relieve symptoms by blocking sensory nerves that cause pain in chronic pain patients [2]. Sweet and Wepsic used radiofrequency on the Gasserian ganglion with a percutaneous approach for the treatment of trigeminal neuralgia [3]. Nugent and Berry described detailed surgical methods for the treatment of trigeminal neuralgia based on radiofrequency coagulation [4]. Since then, its application has been widened to include motor nerves. Kasdon and Lathi used radiofrequency for the treatment of posttraumatic spasticity [5].

At the early stage of development, masseter muscle reduction was performed by coagulating and causing necrosis of the involved masseter muscle using radiofrequency [2]. 
Afterwards the procedure has developed into a type of neurectomy that blocks nervemuscle communication through the application of radiofrequency with a nerve stimulator device that can search for motor nerves within the muscle [2]. Compared to electric cautery which is done with electrosurgical devices and surgical lasers that directly transmit high output heat to destroy tissue, alternating current radiofrequency devices induce ionic agitation to the tissue, which continually generates frictional heat in the tissue itself. The tissue is coagulated by this secondary heat energy [6].

A previous paper reported that side-effects due to muscle tissue damage were less with radiofrequency neurectomy since the technique only thermally coagulates motor nerves innervating the muscle itself and the therapeutic duration is longer compared to that resulting from botulinum toxin injection. However, related reports are relatively sparse in spite of the increasing number of complaints of side-effects after masseteric nerve neurectomy [2].

Therefore, in this case series we will present 2 cases consisting of various symptoms in relation to the radiofrequency neurectomy procedure that was done on the masseteric nerve, along with other relevant clinical characteristics. The case series also includes a literature review on the few studies that report on radiofrequency neurectomy of the masseteric nerve. The details provided in this article will help clinicians to understand possible side-effects that are specific to the procedure and distribute measures to be taken during the diagnostic process for appropriate patient selection and prevention and alleviation of possible side-effects including aggravation of TMD symptoms.

\section{Case Series}

\subsection{Case 1}

A 21-year-old male patient visited the orofacial pain clinic of our dental hospital with the chief complaint of complications occurring after masseteric nerve neurectomy using radiofrequency which he had received 11 days ago. He had been suffering from neck and shoulder pain since 4 years ago. Starting from 3 weeks ago, he experienced the pain intensifying when he was trying to concentrate on his work. Two weeks ago, he visited an orthopedic clinic and had magnetic resonance imaging (MRI) of the cervical area. The orthopedic surgeon confirmed that there was no disc disorder of the neck. Eleven days ago, he visited a private dental clinic and was diagnosed with disc displacement of the temporomandibular joint (TMJ) after clinical and radiographic examinations. Joint sounds were present on mouth opening at that time. Following diagnosis, masseteric nerve neurectomy was suggested as treatment and conducted. One week ago the patient experienced a decrease in the intensity of neck and shoulder pain after the treatment. When he first visited our clinic, the patient complained of swelling of both cheeks, dizziness, and generalized lethargy.

\subsubsection{Clinical Examinations}

Clinical examinations were based on the Diagnostic Criteria for TMD (DC/TMD) [7]. Mouth opening limitation was observed along with local heat and mild swelling in both masseter muscle areas. Comfortable mouth opening (measured between the incisal edge of the upper and lower central incisors and the amount of overbite added) was $30 \mathrm{~mm}$ and maximum mouth opening was $33 \mathrm{~mm}$ accompanied by pain in both masseter muscles. The mandibular movement path showed S-shaped deviation on mouth opening. The patient did not report pain on palpation of the TMJ capsule area. When masticatory and cervical muscles were palpated the patient reported tenderness of both masseters, the left sternocleidomastoid, the left trapezius, and the left splenius capitis muscle. The patient also reported pain of the left TMJ area when performing eccentric movements to the right. He reported parafunctions including clenching, enjoying hard food, unilateral sleep, indigestion, and bad body posture. He was undergoing orthodontic treatment to correct malocclusion which had started before the neurectomy procedure. Examination of the occlusion showed all premolar and molar teeth firmly occluding in maximum intercuspal 
position. The upper first premolars of both sides had been extracted for orthodontic reasons. Blood tests with complete blood cell count and differential and blood chemistry showed an increase in segmental neutrophil, absolute neutrophil count, and total protein, while showing a decrease in lymphocyte and eosinophil counts. The erythrocyte sedimentation rate was within normal range. All subcategory scores of the Symptom Checklist-90-Revised (SCL-90-R) were within normal range [8].

\subsubsection{Imaging}

On plain radiographs including panoramic, TMJ panoramic, and transcranial views, no significant bony changes of the condyles were observed, but the movement of both condyles appeared to be restricted (Figure 1).
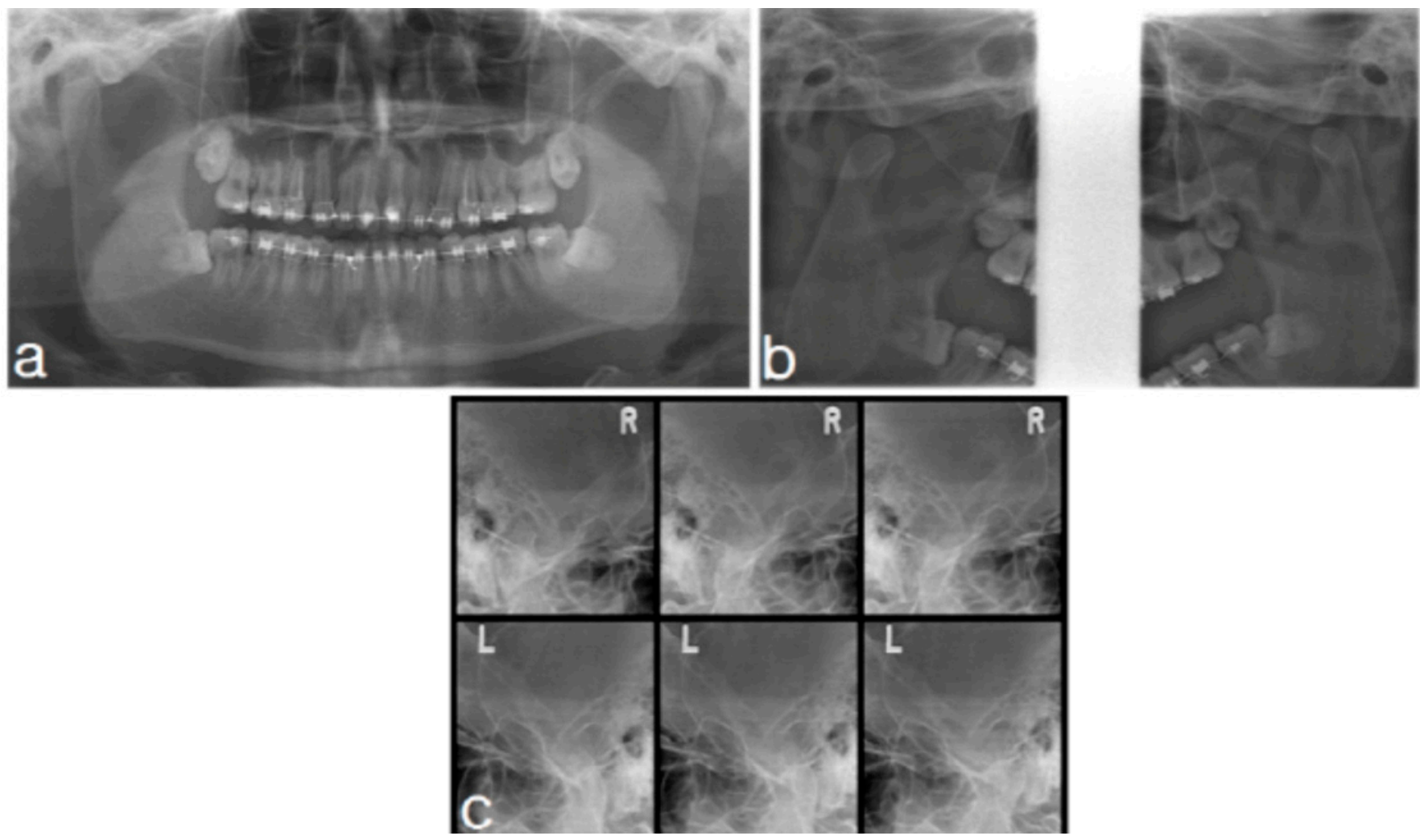

Figure 1. Plain radiographs taken at the first visit. Limited movement of both condyles. (a): panoramic view; (b): TMJ panoramic view; and (c): transcranial view.

TMJ MRI was performed to rule out the possibility of inflammatory myositis. The position of both articular discs was normal at both closed and open mouth positions and the condyles showed sufficient translation to be positioned directly below the articular eminence at the time of mouth opening (Figure 2).

However, heterogeneous hyperintense T2 signals were observed within the masseter muscle of both sides (Figure 3). Based on such findings the patient was diagnosed as having post-procedural inflammation with possible hemorrhage of the masseter muscle. 

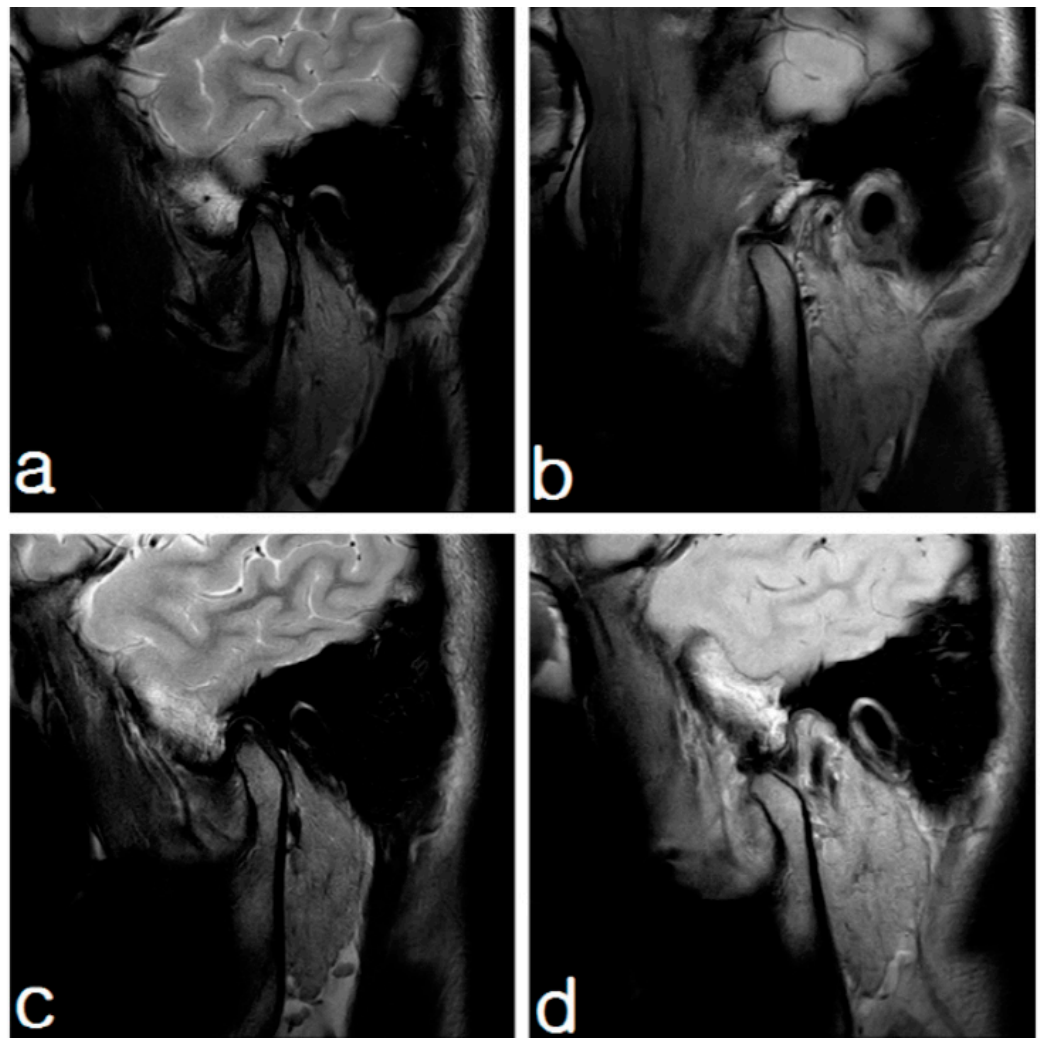

Figure 2. Temporomandibular joint magnetic resonance imaging (proton density, sagittal view). The positions of both articular discs were normal at both closed and open mouth positions. (a): left, closed; (b): left, open; (c): right, closed; and (d): right, open.

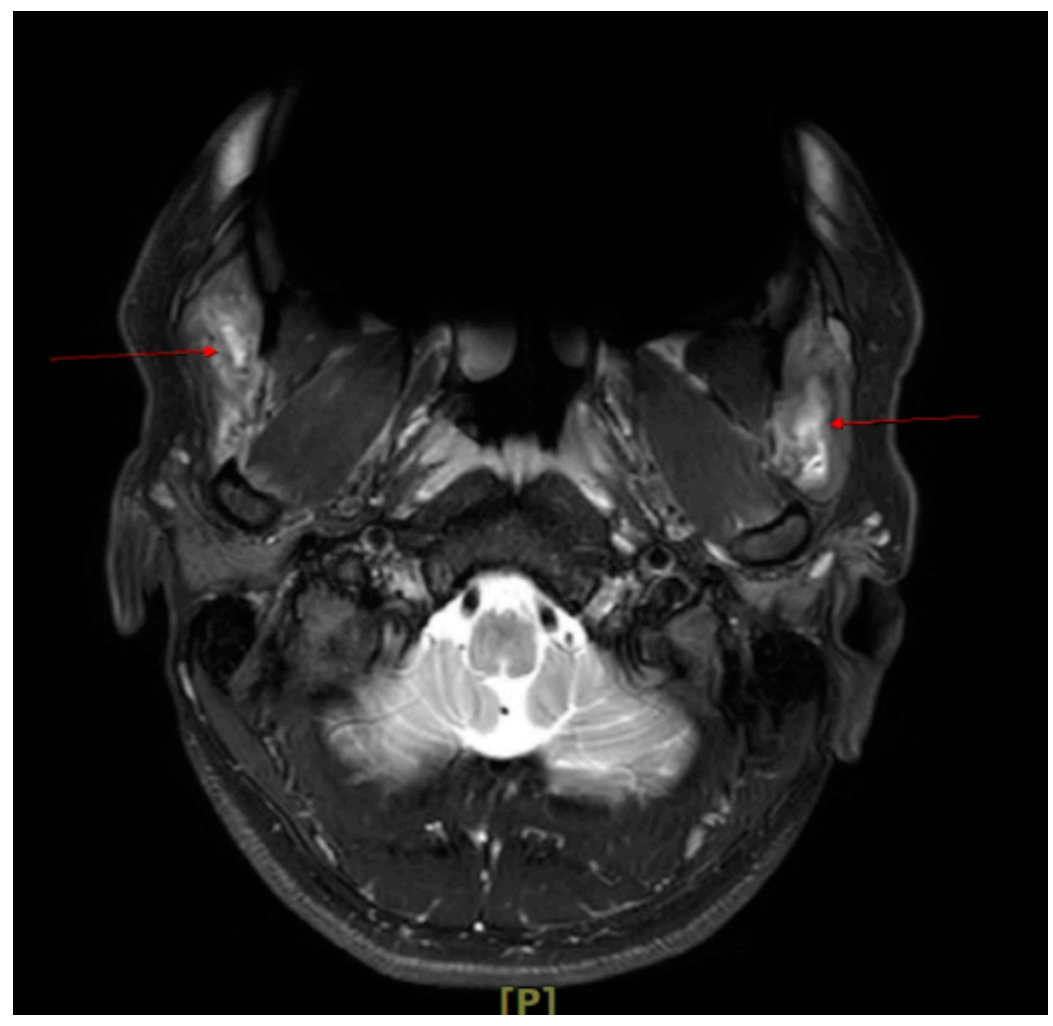

Figure 3. Temporomandibular joint magnetic resonance imaging (axial view). 
Heterogeneous hyperintense T2 signals were observed within the masseter muscle of both sides (arrows).

\subsubsection{Treatment}

At the first visit, antibiotics (Augmentin $625 \mathrm{mg} 3$ times a day for 10 days) and analgesics (Zaltoprofen $80 \mathrm{mg} 3$ times a day for 10 days) were prescribed based on the possibility of infection following the invasive treatment. After 1 week, the amount of mouth opening returned to normal range with partial relief of symptoms, but mild heat could still be detected on both masseter muscles. Comfortable mouth opening was $38 \mathrm{~mm}$ and maximum mouth opening was $40 \mathrm{~mm}$. The patient reported that symptoms of subjective dizziness, systemic lethargy, and facial swelling were reduced. However, pain was still present when both masseter and temporalis muscles were palpated. Physical therapy including moist hot pack application and transcutaneous electrical nerve stimulation (TENS, $10-15 \mathrm{MHz}$ for $45 \mathrm{~min}$ ) was conducted on both masseter muscles and the patient was trained to apply moist heat on the affected area 3 times a day along with $6 \times 6$ exercises [9] as a home-based protocol with the objective of further pain improvement. Behavioral therapy for the correction of parafunctional habits was also instructed. Once again antibiotics and analgesics were prescribed with the same regimen as the first prescription for an additional 10 days. When the patient returned after 2 weeks, symptoms were further relieved and increased heat on the masseter muscles was no longer present. The patient reported that the dizziness and lethargy he had been experiencing had been further reduced and the swelling of the masseter area had totally disappeared. Comfortable mouth opening remained at $38 \mathrm{~mm}$ and maximum mouth opening was $40 \mathrm{~mm}$. The patient still reported pain when both masseter and temporalis muscles were palpated. Physical therapy including moist heat, TENS, and low level laser therapy (LLLT, red diode laser at wave length $650 \pm 20 \mathrm{~nm}$ for $10 \mathrm{~min}$ ) was conducted [10]. The patient was instructed to regularly practice physical therapy at home and revisit if symptoms worsened.

\subsection{Case 2}

A 28-year-old male patient visited the orofacial pain clinic of our dental hospital with the chief complaint of occlusal discomfort which he described as a sensation of the mandible being positioned forward and teeth not occluding properly. He had persistently felt waxing and waning stiffness and discomfort in the left TMJ area when chewing. He also reported neck and shoulder pain which had started 2 years ago. The patient visited a private dental clinic 1 year ago for the treatment of symptoms occurring in the TMJ area. At that point masseteric nerve neurectomy with radiofrequency was suggested and subsequently conducted. He had experienced occlusal discomfort following the procedure.

\subsubsection{Clinical Examinations}

The mouth opening path showed S-shaped deviation. The patient reported tenderness on palpation of the left TMJ capsule and the left temporalis muscle area. The comfortable mouth opening of $53 \mathrm{~mm}$ and maximum mouth opening of $58 \mathrm{~mm}$ were within normal range. However, the occlusion was abnormal with only both second molars occluding. Anterior open bite was observed with an overbite of $-1 \mathrm{~mm}$. Both masseter muscles showed atrophy. He reported parafunctional habits such as tooth grinding and clenching, frequent intake of hard food, unilateral chewing and sleep, using a hard pillow, irregular diet, indigestion, and bad body posture. SCL-90-R results showed a high score $(\geq 60)$ in 7 subcategories (somatization 61, obsessive-compulsive 67, interpersonal sensitivity 66 , depression 60, phobic anxiety 66, global severity index 62, positive symptom total 68). Hematologic test results including complete blood cell count with differential and blood chemistry were within normal limits. 


\subsubsection{Imaging}

On the TMJ cone-beam computed tomography (CBCT) taken 6 months before the first visit to our clinic, the right TMJ condyle appeared to show signs of incipient degenerative change and the left condyle appeared to be at a more advanced stage of degenerative disease with signs of cortical erosion (Figure 4).
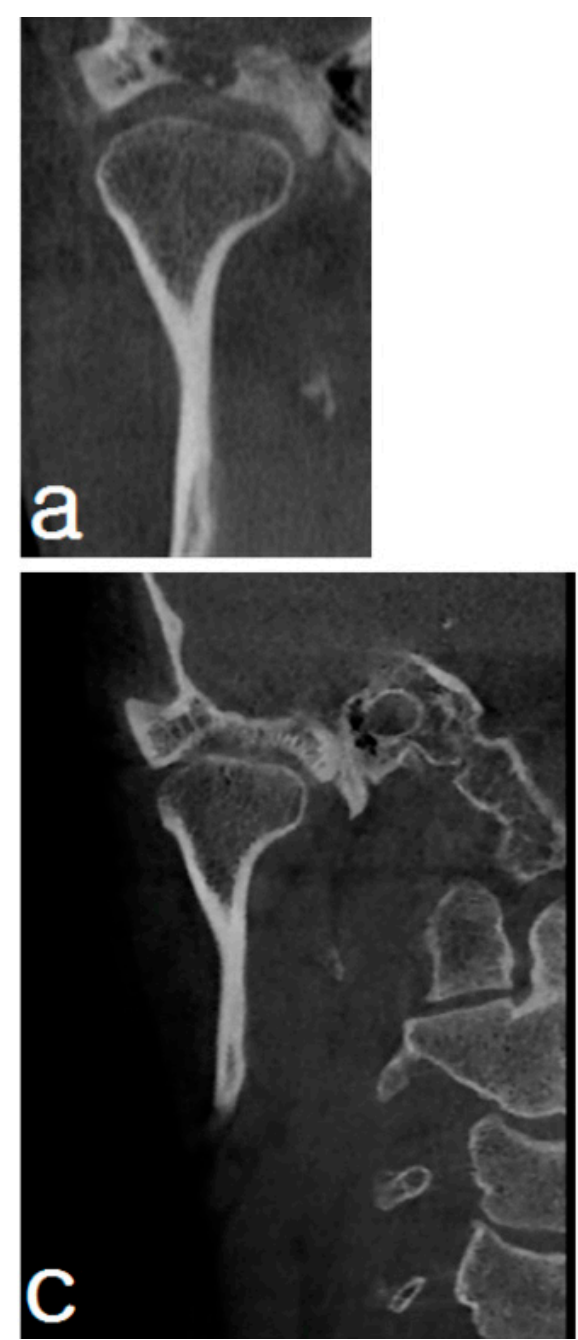
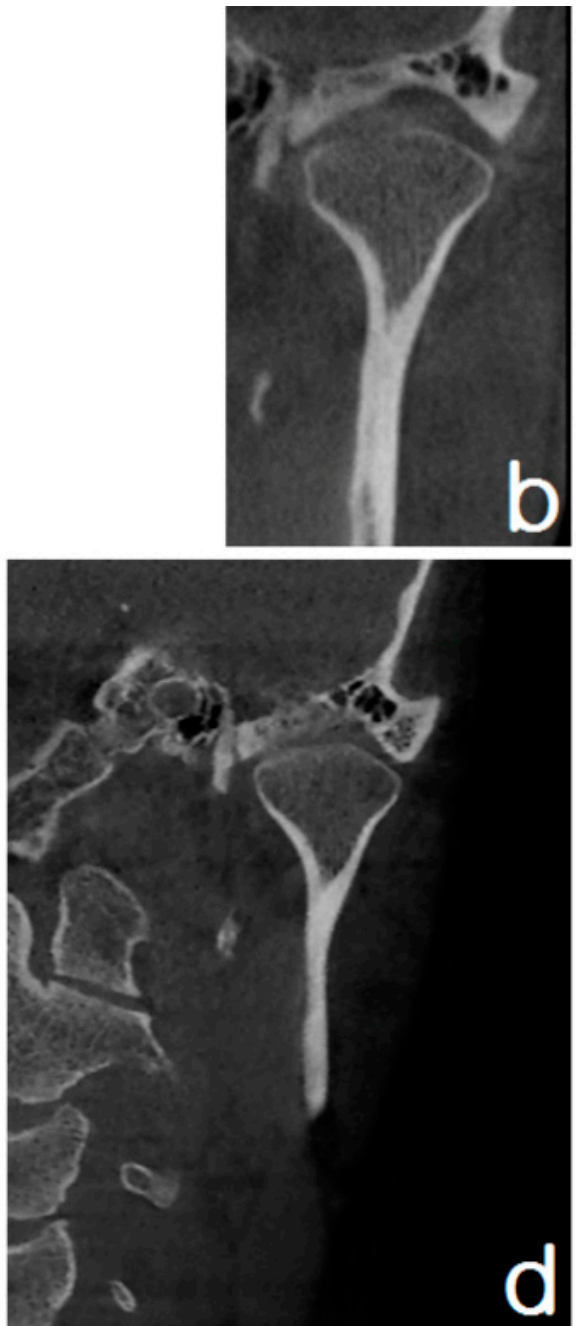

Figure 4. Temporomandibular joint cone beam computed tomography. (a): taken 6 months before the first visit, right condyle, incipient degenerative change; (b): taken 6 months before the first visit, left condyle, advanced stage of degenerative change; (c): taken 8 months after treatment, right condyle, no further destructive changes appeared; and (d): taken 8 months after treatment, left condyle, no further destructive changes appeared.

Plain radiographs taken at the time of the patient's first visit showed incipient degenerative change on the left condyle (Figure 5). On the CBCT and plain radiographs taken 8 months later following treatment, no further destructive change was observed (Figure 4). Based on the initial imaging results and clinical examination, the patient was diagnosed as having degenerative joint disease of the TMJ. 

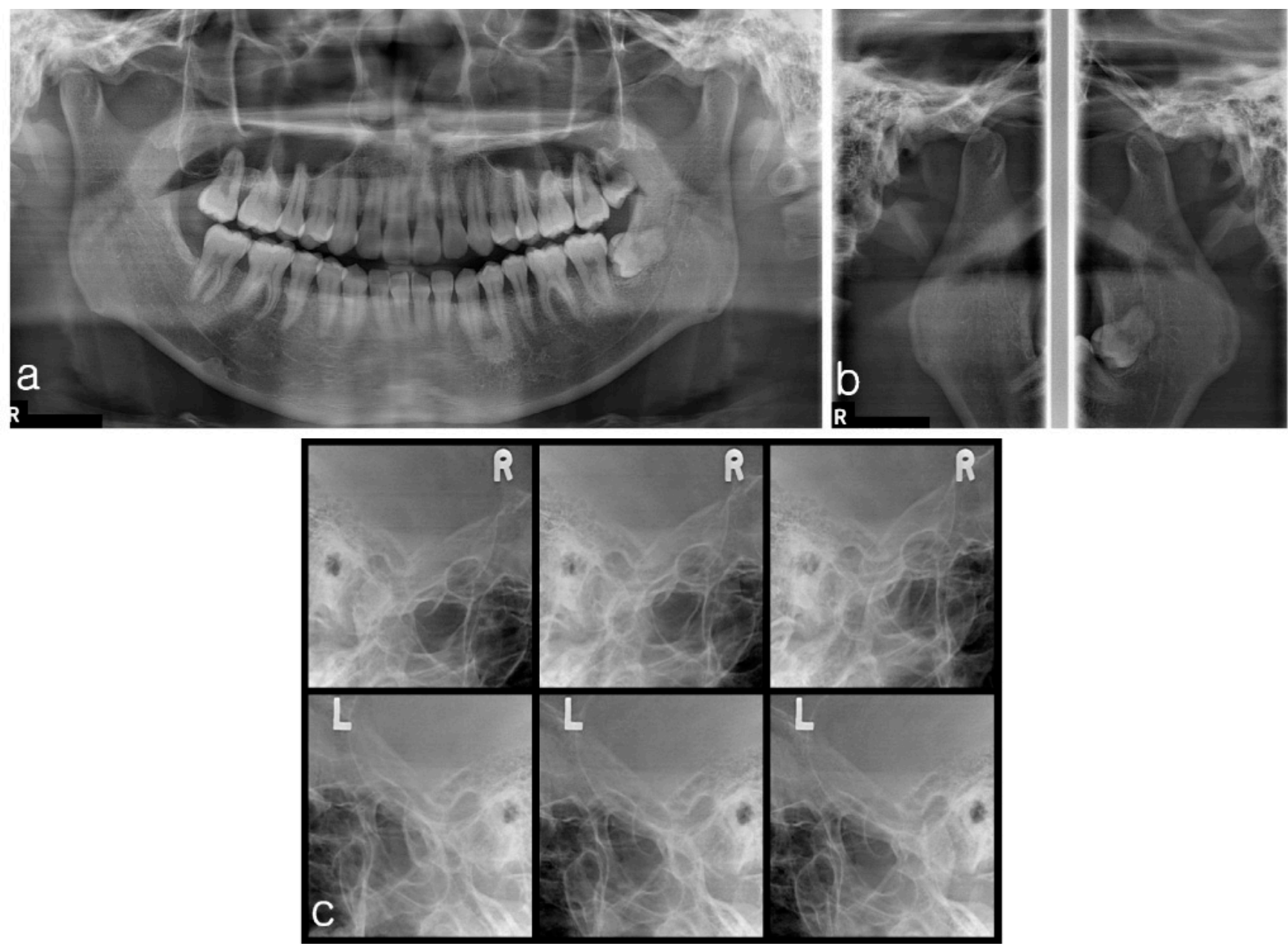

Figure 5. Plain radiographs taken at first visit. Incipient degenerative change on the left condyle. (a): panoramic view; (b): TMJ panoramic view; and (c): transcranial view.

\subsubsection{Treatment}

At the first visit, he received physical therapy including moist hot pack therapy on the masseter muscles and was trained to apply moist heat and do $6 \times 6$ exercises as a home-based protocol on a regular basis. After 1 month of physical and behavioral therapy, his symptoms did not show improvement. For further improvement occlusal stabilization splint therapy was initiated at that point. The splint was a custom-made appliance built with hard resin to cover the occlusal surface of all upper teeth and the patient was instructed to wear it 8 to $10 \mathrm{~h}$ a day including during sleep. At the time of splint insertion the upper and lower right first and second molars and left second molars occluded. Following 2 weeks of splint therapy, the patient felt that the stiffness in the left TMJ, neck, and shoulder area had decreased and occlusal discomfort was also improving. Examination of occlusion showed that the upper and lower right first and second molars and left second molars occluded. Overbite remained at $-1 \mathrm{~mm}$. When the patient returned 2 months later, he reported a worsening of overall symptoms with stinging pain in both TMJ areas and stiffness of the neck, left shoulder, waist, and pelvic areas. Comfortable and maximum mouth opening was $50 \mathrm{~mm}$. Both upper and lower second molars occluded. During the follow-up period of 8 months with treatment, his symptoms repeatedly fluctuated. Occlusion showed little improvement (Figure 6). Eight months after the first visit, symptoms were generally stabilized and alleviated. He continues to undergo treatment every two months with splint adjustment and physical therapy including moist 
heat, ultrasound ( $3 \mathrm{~W} / \mathrm{cm}^{2}, 1 \mathrm{MHz}$ for $10 \mathrm{~min}$ ), LLLT, and TENS therapy (identical protocol as in case 1$)$.

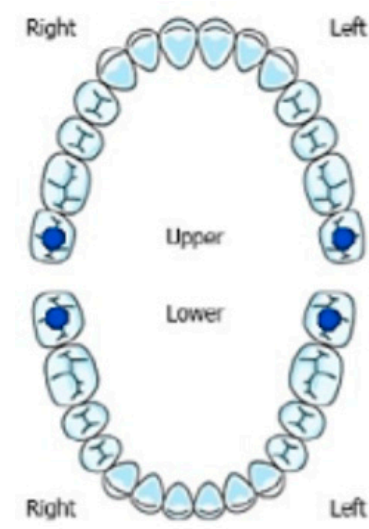

1 st visit

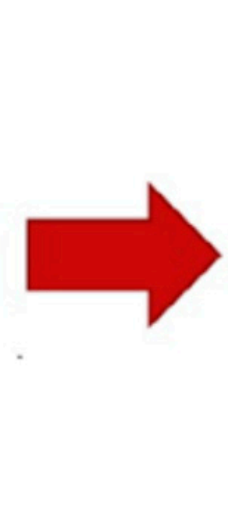

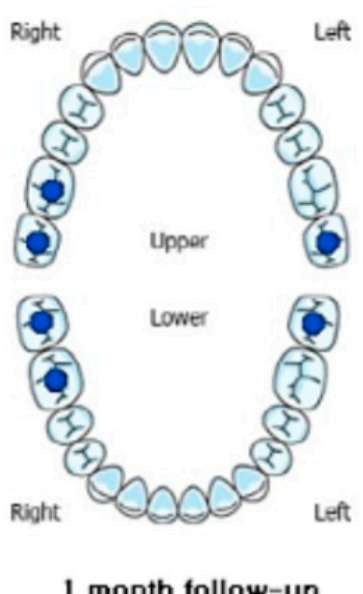

1 month follow-up

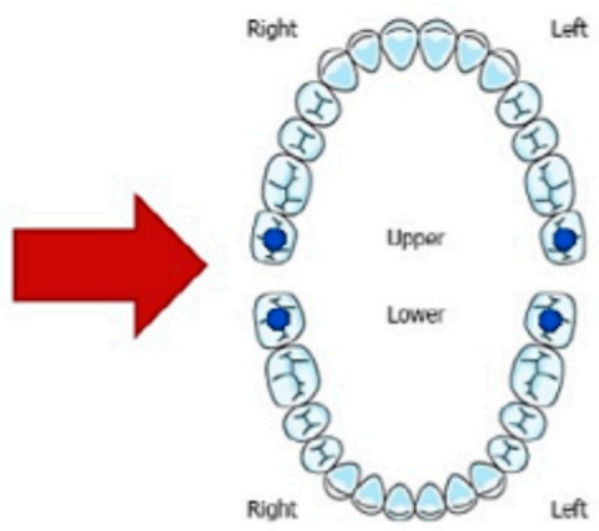

3.5 months follow-up

Figure 6. Occlusion status.

At the first visit, only both second molars occluded. One month later, the upper and lower right first and second molars and left second molars occluded. Two months and two weeks later, only both second molars occluded. At the latest visit, occlusion did not show further change.

\section{Discussion}

TMD is characterized by the presence of symptoms including pain, TMJ noises and restricted mandibular movement. The etiology of TMD is known to be multifactorial and includes biological, environmental, and psychosocial factors [11]. Conventional treatment for TMD includes conservative therapies such as behavioral modification, physical therapy, pharmacotherapy, and oral appliances [9]. Previous studies sometimes provide conflicting results regarding their effectiveness according to the treatment approach and investigation methodology. A previous meta-analysis reported that occlusal appliance, certain pharmacologic agents, behavioral therapy, jaw exercises and postural training are effective in alleviating TMD pain [12] and such approaches also efficiently improved sleep quality and quality of life [13]. However, a recent review does not show sufficient evidence to support the usage of topical medication for TMD treatment [14]. A previous meta-analysis concluded that splint therapy can reduce pain related to TMD [15], however another randomized controlled trial did not support its effectiveness in reducing subjective pain [16]. A recent systematic review suggests the possibility of myofunctional therapy as a cost-effective TMD treatment [17]. Unfortunately, there are no well-designed investigations on the effect of masseteric nerve neurectomy with radiofrequency on TMD signs and symptoms.

Radiofrequency has been applied in various muscle areas. Calf reduction is the most commonly conducted procedure and is generally performed in the field of plastic surgery. Radiofrequency based gastrocnemius muscle reduction brought esthetically satisfactory results $[18,19]$. However, muscle contracture due to excessive muscle fibrosis was reported as a side-effect [20]. Another procedure done with radiofrequency is glabellar frowning reduction by ablation of efferent branches of the temporal and angular nerves; the procedure efficiently eliminates corrugator and procerus muscle contraction [21,22].

Radiofrequency ablation has also been applied for the reduction of pain. Ablation targeting preganglionic fibers of the Gasserian ganglion has been done to treat trigeminal neuralgia [3,4]. A study based on 1600 trigeminal neuralgia patients showed pain relief in $92 \%$ of the patients 5 years after the first ablation was performed. Side-effects included diminished corneal reflex, masseter muscle weakness and paralysis, dysesthesia, anesthesia 
dolorosa, keratitis, and transient paralysis of the third and fourth cranial nerves [23]. In case of complications related to direct nerve injury, type I collagen-based devices may be considered as an alternative to conventional microsurgery with autologous grafts [24].

Reports related to the masseter muscle area are relatively sparse. Masseter muscle reduction using radiofrequency coagulation has been generally conducted with the objective of reducing masseter muscle hypertrophy. The procedure resulted in a $10-60 \%$ (mean 27\%) reduction in muscle volume at 6-month post-operative follow-up, with most patients satisfied with the esthetic improvement [25]. Another study also reported pronounced reduction of masseter muscle volume [26]. The reported side-effects included mucoserous fluid collection, persistent mouth opening limitation, delayed bleeding, infection, long-lasting pain, facial nerve injury, and parotid gland injury. Mouth opening limitation subsided in most cases with time except for 1 case that presented with symptoms lasting even after 10 months [25].

In a study based on 19 female patients whose chief complaint was masseter muscle hypertrophy and who received masseteric nerve neurectomy, post-operative swelling or pain was found in all patients but the degree was mild and the symptoms resolved within 1-2 weeks in most cases. A decrease in masticatory function was observed after the procedure in all patients, but there were no patient complaints related to it. Pain on mouth opening was observed in 3 patients but resolved within 1 week. Reduction of the masseter muscle volume was evident after approximately 1 month and 17 out of 19 patients were satisfied with the results [2].

Huang et al. compared the effect of masseter muscle reduction based on radiofrequency and botulinum toxin A injection. Masseter muscle thickness decreased up to 6 months post-treatment but increased afterwards until 12 months following the injection while muscle thickness steadily decreased over 12 months with radiofrequency. Thus, the authors concluded that the effect of masseter muscle reduction using radiofrequency was more significant than that from botulinum toxin A injection [27]. There is only one case study about masseteric nerve neurectomy using radiofrequency for the treatment of masseter muscle hypertrophy [2].

Recently, some clinicians are attempting masseteric nerve neurectomy for the treatment of TMD symptoms. Unfortunately, there is only a single case report that described worsening of myofascial pain following masseteric nerve neurectomy [28].

In case 1, MRI analysis resulted in the diagnosis of inflammatory myositis. Medication and physical therapy were effective in relieving symptoms including mouth opening limitation. From this case, it can be seen that side-effects that may occur with masseter muscle reduction using radiofrequency based coagulation may also occur with masseteric nerve neurectomy. Mouth opening limitation is generally known to resolve over time, but caution is required since cases of permanent mouth opening limitation have been reported [25].

In case 2, it is presumed that the occlusal discomfort may have been caused by asymmetry in the masseter muscles due to the imbalanced application of radiofrequency on each side. Animal study results showed that the degree of masseter muscle reduction was dependent on the number of radiofrequency applications [29]. Another possible cause is the pre-existing degenerative joint disease of the TMJ condyles of this patient; joint disease could be identified on CBCT images taken prior to the first visit to our clinic. Procedures done on the orofacial region may have resulted in increased awareness of a previously existing malocclusion. The prevalence of TMJ degenerative joint disease in adults is $9.8 \%$ [30] and conventional treatment includes occlusal splint, medication, and physical therapy [31]. TENS therapy that blocks nociceptive transmission by activating $A \beta$ fibers is known as an efficient method to treat multifactorial pain such as cervical pain and TMD [32]. Prior to performing masseteric nerve neurectomy, which is an invasive treatment with irreversible results, conservative treatment should have been considered first. Since the effects of masseter muscle reduction are permanent, occlusal adjustment or orthodontic treatment could be considered if necessary, after TMD related symptoms 
are sufficiently relieved. The psychological evaluation scores were high with the patient of case 2 and as chronic pain is commonly associated with psychological conditions such as anxiety and depression that can affect long-term prognosis [8], psychological evaluation of the patient could be another aspect to consider in the evaluation process of a TMD patient for successful treatment results with radiofrequency neurectomy.

Before considering masseteric nerve neurectomy, close and thorough clinical examinations are necessary to avoid poor treatment response and unwanted side-effects. Myogenous TMD constitutes approximately $45 \%$ of all TMD cases [33] and in spite of its high prevalence, the underlying mechanism remains elusive. Evidence shows that masticatory muscle hyperactivity could be irrelevant to the generation of persistent orofacial muscle pain [34]. Additionally, a previous study showed that there was no significant difference in masseter muscle width between myofascial pain patients and pain free controls [35]. Evidence supports the role of a central nociceptive pathway in myogenous pain [36]. Thus, when the severity of pain is high and pain is of a longer duration, it is highly likely that masseteric nerve neurectomy will not produce significant improvements. Hence meticulous evaluation of the TMD patient based on stringent diagnostic criteria should be carried out before considering the patient as a candidate for masseteric nerve neurectomy based on radiofrequency. A previous study reported cases treated with masseteric nerve neurectomy showing unfavorable results as pain and dysfunction continued after the treatment. Nam et al. reported cases of myofascial orofacial pain worsening after masseteric nerve neurectomy with side-effects including mouth opening limitation and open bite [28].

Studies on masseter muscle reduction including those achieved through masseteric nerve neurectomy that have been published to date were limited to those handling masseter muscle hypertrophy and its reduction for cosmetic purposes. There is no case-controlled study on masseter muscle reduction and masseteric nerve neurectomy for the treatment of TMD.

\section{Conclusions}

Based on the above review there is no scientific evidence until now to support the application of masseteric nerve neurectomy for the treatment of TMD. Therefore, conservative treatment still remains the first line approach for TMD and masseteric nerve neurectomy should only be considered when well-designed research results on TMD patients report favorable outcomes that outweigh the possible side-effects of an invasive treatment. Such studies should be able to provide a guideline for patient selection and treatment result evaluation to minimize related side-effects and optimize treatment results. Until then, it would be logical to argue that masseteric nerve neurectomy should be limited to those that require masseter muscle reduction due to muscle hypertrophy for cosmetic purposes.

Author Contributions: Conceptualization, J.W.P. and I.-S.C.; methodology, J.W.P.; investigation, I.-S.C.; writing —original draft preparation, I.-S.C. and J.H.J.; writing—-review and editing, J.H.J. and J.W.P.; visualization, I.S.C.; supervision, J.W.P. All authors have read and agreed to the published version of the manuscript.

Funding: This research received no external funding and the APC was funded by Seoul National University.

Institutional Review Board Statement: The study was conducted according to the guidelines of the Declaration of Helsinki, and approved by the Institutional Review Board of Seoul National University Dental Hospital (protocol code \#ERI 20028, date of approval 29 October 2020).

Informed Consent Statement: Patient consent was waived due to the fact that identifiable information was not included in the study.

Data Availability Statement: The data that support the findings of this study are available on request from the corresponding author. The data are not publicly available due to privacy or ethical restrictions.

Conflicts of Interest: The authors declare no conflict of interest. 


\section{References}

1. Yoon, J.S. Medical Dispute Mediation and Arbitration Case Book 2018/2019; Korea Medical Dispute Mediation and Arbitration Agency: Seoul, Korea, 2020; pp. 439-443.

2. Kwon, J.O.; Lee, M.J.; Lee, K.H.; Choi, M.H. Masseter muscle reduction using radiofrequency rhizotomy. J. Korean Soc. Aesth. Plast. Surg. 2008, 14, 43-48. Available online: https: / /www.ksaps.or.kr/journal/view.php?sid=432 (accessed on 17 June 2021).

3. Sweet, W.H.; Wepsic, J.G. Controlled thermocoagulation of trigeminal ganglion and rootlets for differential destruction of pain fibers Part 1: Trigeminal neuralgia. J. Neurosurg. 1974, 40, 143-156. [CrossRef]

4. Nugent, G.R.; Berry, B. Trigeminal neuralgia treated by differential percutaneous radiofrequency coagulation of the Gasserian ganglion. J. Neurosurg. 1974, 40,517-523. [CrossRef]

5. Kasdon, D.L.; Lathi, E.S. A prospective study of radiofrequency rhizotomy in the treatment of posttraumatic spasticity. Neurosurgery 1984, 15, 526-529. [CrossRef] [PubMed]

6. Sluijter, M.; Racz, G. Technical aspects of radiofrequency. Pain Pract. 2000, 2, 195-200. [CrossRef] [PubMed]

7. Schiffman, E.; Ohrbach, R.; Truelove, E.; Look, J.; Anderson, G.; Goulet, J.P.; List, T.; Svensson, P.; Gonzalez, Y.; Lobbezoo, F.; et al. Diagnostic criteria for temporomandibular disorders (DC/TMD) for clinical and research applications: Recommendations of the International RDC/TMD Consortium Network and Orofacial Pain Special Interest Group. J. Oral Facial Pain Headache 2014, 28, 6-27. [CrossRef]

8. Ko, M.Y. Psychological analysis of TMD patients through the SCL-90-R. J. Oral Med. Pain 1999, $24,59-67$.

9. Kim, C. Conservative treatment modalities for patients with temporomandibular joint (TMJ) disorders. J. Korean Dent. Assoc. 2013, 51, 74-83.

10. Kim, J.H.; Park, J.S.; Ko, M.Y. The effects of low level laser therapy on current perception threshold in orofacial region. J. Oral Med. Pain 2002, 27, 391-400.

11. Dworkin, S.F.; Von Korff, M.R.; LeResche, L. Epidemiologic studies of chronic pain: A dynamic-ecologic perspective. Ann. Behav. Med. 1992, 14, 3-11. [CrossRef]

12. List, T.; Axelsson, S. Management of TMD: Evidence from systematic reviews and meta-analyses. J. Oral Rehabil. 2010, 37, 430-451. [CrossRef]

13. de Resende, C.; de Oliveira Medeiros, F.; de Figueiredo Rêgo, C.R.; Bispo, A.; Barbosa, G.; de Almeida, E.O. Short-term effectiveness of conservative therapies in pain, quality of life, and sleep in patients with temporomandibular disorders: A randomized clinical trial. Cranio 2019, 1-9. [CrossRef]

14. Mena, M.; Dalbah, L.; Levi, L.; Padilla, M.; Enciso, R. Efficacy of topical interventions for temporomandibular disorders compared to placebo or control therapy: A systematic review with meta-analysis. J. Dent. Anesth. Pain Med. 2020, 20, 337-356. [CrossRef]

15. Ebrahim, S.; Montoya, L.; Busse, J.W.; Carrasco-Labra, A.; Guyatt, G.H. The effectiveness of splint therapy in patients with temporomandibular disorders: A systematic review and meta-analysis. J. Am. Dent. Assoc. 2012, 143, 847-857. [CrossRef]

16. Deregibus, A.; Ferrillo, M.; Grazia Piancino, M.; Chiara Domini, M.; de Sire, A.; Castroflorio, T. Are occlusal splints effective in reducing myofascial pain in patients with muscle-related temporomandibular disorders? A randomized-controlled trial. Turk. J. Phys. Med. Rehabil. 2021, 67, 32-40. [CrossRef]

17. Melis, M.; Di Giosia, M.; Zawawi, K.H. Oral myofunctional therapy for the treatment of temporomandibular disorders: A systematic review. Cranio 2019, 17, 1-7. [CrossRef]

18. Park, Y.J.; Jo, Y.W.; Bang, S.I.; Kim, H.J.; Lim, S.Y.; Mun, G.H.; Hyon, W.S.; Oh, K.S. Radiofrequency volume reduction of gastrocnemius muscle hypertrophy for cosmetic purposes. Aesth. Plast. Surg. 2007, 31, 53-61. [CrossRef] [PubMed]

19. Lee, A. Calf reduction using radiofrequency (RF) directed to the gastrocnemius muscle. J. Korean Soc. Aesth. Plast. Surg. 2007, 13, 105-110. Available online: https:/ / www.ksaps.or.kr/journal/view.php?sid=399 (accessed on 17 June 2021).

20. Hwang, C.H. Excessive gastrocnemius fibrosis developed after radiofrequency-induced cosmetic volume reduction. Aesth. Plast. Surg. 2011, 35, 1172-1175. [CrossRef]

21. Foster, K.W.; Fincher, E.F.; Moy, R.L. Radiofrequency ablation of facial nerve branches controlling glabellar frowning. Dermatol. Surg. 2009, 35, 1908-1917. [CrossRef] [PubMed]

22. Newman, J. Radiofrequency (GFX ${ }^{\mathrm{TM}}$ ) ablation for the reduction of glabellar frowning. Facial Plast. Surg. 2010, 26, 266-273. [CrossRef]

23. Kanpolat, Y.; Savas, A.; Bekar, A.; Berk, C. Percutaneous controlled radiofrequency trigeminal rhizotomy for the treatment of idiopathic trigeminal neuralgia: 25-year experience with 1,600 patients. Neurosurgery 2001, 48, 524-534. [CrossRef] [PubMed]

24. Roccuzzo, A.; Molinero-Mourelle, P.; Ferrillo, M.; Cobo-Vázquez, C.; Sanchez-Labrador, L.; Ammendolia, A.; Migliario, M.; de Sire, A. Type I collagen-based devices to treat nerve injuries after oral surgery procedures. A systematic review. Appl. Sci. 2021, 11, 3927. [CrossRef]

25. Park, Y.J.; Jo, Y.W.; Bang, S.I.; Kim, H.J.; Lim, S.Y.; Mun, G.H.; Hyon, W.S.; Oh, K.S. Radiofrequency volumetric reduction for masseteric hypertrophy. Aesth. Plast. Surg. 2007, 31, 42-52. [CrossRef] [PubMed]

26. Ham, J.W. Masseter muscle reduction procedure with radiofrequency coagulation. J. Oral Maxillofac. Surg. 2009, 67, 457-463. [CrossRef] [PubMed]

27. Huang, J.L.; Chen, G.; Chen, X.D.; Zhou, B.R.; Luo, D. A comparative study of the efficacy and safety of radiofrequency ablation and botulinum toxin A in treating masseteric hypertrophy. Exp. Ther. Med. 2014, 7, 1203-1208. [CrossRef] [PubMed] 
28. Nam, H.; Ko, D.; Kang, J.K.; Shim, Y.J. Myofascial orofacial pain exacerbated after masseteric nerve neurectomy. J. Oral Med. Pain 2020, 45, 110-114. [CrossRef]

29. Song, K.H.; Kim, M.H.; Jung, J.W.; Kim, A.S.; Hong, S.P.; Kim, S.G. The change in dimension of the masseter muscle in rabbits after radiofrequency therapy. J. Oral Maxillorfac. Surg. 2009, 67, 485-490. [CrossRef]

30. Valesan, L.F.; Da-Cas, C.D.; Reus, J.C.; Denardin, A.C.S.; Garanhani, R.R.; Bonotto, D.; Januzzi, E.; de Souza, B.D.M. Prevalence of temporomandibular joint disorders: A systematic review and meta-analysis. Clin. Oral Investig. 2021, 25, 441-453. [CrossRef]

31. Tanaka, E.; Detamore, M.S.; Mercuri, L.G. Degenerative disorders of the temporomandibular joint: Etiology, diagnosis, and treatment. J. Dent. Res. 2008, 87, 296-307. [CrossRef]

32. Paolucci, T.; Agostini, F.; Paoloni, M.; de Sire, A.; Verna, S.; Pesce, M.; Ribecco, L.; Mangone, M.; Bernetti, A.; Saggini, R. Efficacy of TENS in cervical pain syndromes: An umbrella review of systematic reviews. Appl. Sci. 2021, 11, 3423. [CrossRef]

33. Manfredini, D.; Guarda-Nardini, L.; Winocur, E.; Piccotti, F.; Ahlberg, J.; Lobbezoo, F. Research diagnostic criteria for temporomandibular disorders: A systematic review of axis I epidemiologic findings. Oral Surg. Oral Med. Oral Pathol. Oral Radiol. Endod. 2011, 112, 453-462. [CrossRef] [PubMed]

34. Benoliel, R.; Svensson, P.; Heir, G.M.; Sirois, D.; Zakrzewska, J.; Oke-Nwosu, J.; Torress, S.R.; Greenberg, M.S.; Klasser, G.D.; Katz, J.; et al. Persistent orofacial muscle pain. Oral Dis. 2011, 17, 23-41. [CrossRef] [PubMed]

35. Poveda-Roda, R.; Moreno, P.; Bagan, J.; Margaix, M. Myofascial pain: Ultrasound width of the masseter muscle. J. Oral Facial Pain Headache 2018, 32, 298-303. [CrossRef] [PubMed]

36. Sarlani, E.; Grace, E.G.; Reynolds, M.A.; Greenspan, J.D. Evidence for up-regulated central nociceptive processing in patients with masticatory myofascial pain. J. Orofac. Pain 2004, 18, 41-55. 